\title{
Aspects of quality assurance and the link with medical education in the United States
}

\author{
Peter Wilkinson
}

Ashford Hospital, Ashford, Middlesex TW15 3AA, UK

\section{Introduction}

The diverse and continually changing medical scene in the United States, based historically on a free market model, has been accompanied by rapidly increasing costs, often at twice the rate of general inflation. ${ }^{1,2}$ Successive governments have vainly attempted to control these costs and have found it necessary to monitor quality of care as health care providers, faced in certain sectors with a desire to maximize profits, have tried to cut corners. Hospitals and doctors, particularly in the areas where government is paying for care, have found their activities increasingly subject to review by outside agencies. Some view this as harrassment but most have become resigned to it. Quality assurance has therefore arrived in the US on the back of cost control and its associated large bureaucracy. Has the process improved the quality of medical care and has it managed to feed back its findings into medical education? These questions will be examined from the viewpoint of a Health Maintenance Organization (HMO), a hospital and a Peer Review Organization (PRO).

\section{A Health Maintenance Organization ${ }^{3}$}

These organizations have become a model for the British government's purchasing authorities under the White Paper. Doctors contract to work for an HMO on a salaried basis with referrals made through a primary care physician to specialists or hospitals that are part of the scheme. Patients benefit as it is cheaper but they lose the somewhat mythical advantage of being able to choose their own doctor or hospital. In one progressive HMO I visited, quality assurance (QA) was treated scrupulously with an annual budget of \$1 million and a staff of 26 for the programme. Each of the

Correspondence: P.R. Wilkinson, M.D., F.R.C.P. Dr Wilkinson was the National Association of Clinical Tutors Wyeth Travelling Fellow to the USA for 1990 Accepted: 6 September 1990 centres to whom they contracted work had their own QA worker and part-time QA physician. A representative from each of the centres sat on a central QA committee and the quality of care was investigated both at random and for specific conditions. Information was fed back to the centres where remedial action and education were taken to correct problems. If the centre did not respond, for example, if the rate of caesarian section continued to be higher than was thought to be medically justified, the HMO had the sanction of taking their patients elsewhere.

This HMO set particular store in assessing the views of its patients. Someone from the organiza tion regularly phoned its centres pretending to be patient to see how quickly and courteously the phone was answered, and how long the wait was for an appointment. They also sent out questionnaires or phoned the patients to see how they had felt as a result of the medical encounter. For example, would they see the same doctor again? These, and many other questions, were tabulated into an annual report where each centre would see its performance compared with the others, the latter being presented anonymously. Evaluation of outcome measures seemed to be in its infancy.

\section{The hospital scene}

Medical care for the over $65 \mathrm{~s}$ is mainly covered by the Federal Government through Medicare and in order to look after this large group of patients, hospitals have to be accredited. The accreditation process, which covers each participating hospital every 3 years, began in earnest in the early 1950s and to be approved hospitals must have a detailed QA programme which has to include a check on the credentials of their physicians and monitoring of their performance. Quality assurance is therefore an essential component of the life of most hospitals in the US. As well as the statutory requirement demanding QA activities, hospitals see the process as constructive, particularly as patients rapidly 
seek recourse to litigation if they perceive a problem in care. It is felt that QA, by improving the standards of care, will reduce the risk of patients taking legal action and as some hospitals are covering their own legal liabilities they have a strong motivation for limiting any claim. Health authorities in the UK are now covering the costs of all their staff including doctors and are in an analogous situation.

American hospitals have a number of interlinking departments to cover these needs, of which QA is set apart by its need for strict confidentiality and immunity from the law. A large hospital will have a utilization review department checking on whether care is appropriate and efficient according to diagnostic related groups, a legal department which in larger hospitals may contain several lawyers who advise on court cases and damage limitation, and a risk management department. Apparent clinical errors are fed back to the latter department where a plan is developed on how to handle the problem and minimize risk to the individual and the hospital. The collation of problems in such a central department may highlight a practice or piece of equipment which needs alteration.

At a departmental level meetings are held to discuss various issues in a similar fashion to the UK, but in the US there is a clearer path through which personal problems are reported back to the management committees of the hospital where action can be taken. For individual doctors some hospitals may take a firm stance on who can do what and may make doctors who want to perform new procedures go on a formal training programme rather than adopt an attitude of 'see one, do one' and will then monitor their early results. If a particular doctor's performance falls below acceptable standards, the departmental chairman may have the authority to demand more training, more supervision and ultimately the right to remove that doctor from the hospital if matters do not improve. This is made easier than in the UK as many doctors are on 2 year contracts, although, because a doctor may sue for unjust dismissal in the US, the hospital needs to be sure that the case is clearcut. These delicate issues seem to be handled best in the larger academic hospitals, but in the smaller community hospitals where there may only be a few specialists, the influence of personal relationships and the need to maintain referral patterns to perpetuate income can have a bearing on how far matters are taken.

\section{Peer Review Organizations}

In the late 1960s Professional Standards Review Organizations (PSROs) were set up to monitor
Medicare and Medicaid. These were utilization reviews, directed by physicians and aimed at cost control and decreasing variation in standards of care between hospitals. Thirty thousand such organizations, varying between states and institutions, were created, and were then phased out in the early 1980 s by President Reagan. A powerful pressure group, the American Association of Retired People, representing about 50 million citizens, was a major force in ensuring that the quality of care continued to be monitored and the PSROs were thus replaced by the PROs. ${ }^{4}$ Each state had to appoint a PRO and any organization could bid for the contract which was awarded every 2 years.

Initially the PROs concentrated on cost containment with information fed to them through the billing process. A copy of the computer tape related to billing for Medicare would be sent to the PRO where a random $3 \%$ of cases would be selected for review followed by an additional $17 \%$ of cases covering topics that the PRO thought were problem areas. For example neonates are not covered by Medicare but the PRO would find that they were being sent bills for neonatal care. Other items were related to premature discharge, readmissions within 7 days, and transfers to other hospitals.

The quality objectives of PROs were initially defined as follows:

1. Reduce unnecessary hospital readmissions resulting from substandard care during the prior admission.

2. Ensure the provision of medical services without which there is potential for serious complications.

3. Reduce avoidable deaths.

4. Reduce unnecessary surgery or other invasive procedures.

5. Reduce avoidable postoperative or other complications.

The records of those patients picked out for further analysis would then be supplied by the hospital or doctor to a specially trained nurse who would look at the notes using a generic screen in a nonjudgemental fashion. If a problem with quality of care became apparent the notes would be passed to a physician who would look at the points raised by the nurse but who was not restrained by a list of criteria. If a problem was confirmed the notes would be sent to another doctor who would inform the initial doctor giving the physician 30 days to reply.

There are 3 levels of severity for a confirmed problem and if a physician goes over certain thresholds a panel of 3 physicians and a specialist will review all the cases. The panel can send a letter detailing the problem and ask for a return letter from the doctor recognizing the problem with a commitment to change. They can provide appro- 
priate literature references, have a consultant participate in specific cases or require that the doctor completes a certain number of continuing education hours, which can be general or focused on the problem. They may also intensify the review so that all future cases are examined instead of only a proportion thus allowing them to monitor the educational impact of the recommended measures. Sanctions, which are imposed only if there has been a gross and flagrant violation, can include monetary penalties, referral to the State Board or the National Data Bank. The latter has recently been set up to stop doctors who had a problem in one state going across a state border and setting up a new practice. Hospitals will be obliged to report to the Data Bank malpractice payments within 30 days or any reduction in clinical privileges within 15 days. They will also have to query the Data Bank whenever a doctor applies for a position on the staff. If doctors feel that they disagree with the information they have the right to dispute it, but the final arbiter is the Secretary of Health and Human Services. Within the European Economic Community, what safeguards are there to prevent bad doctors moving their clinical practice from one country to another?

The PRO is involved in education. The director of one regularly visited the hospitals in his state to discuss problems highlighted by the organization. However, there did not seem to be any close link with the issues thrown up by the PRO and the formal postgraduate educational programme, perhaps because the PRO was seen at present to be more punitive than constructive. I suspect that this attitude will slowly change. The massive amount of information that the PRO has acquired on doctors means that they are able to track individuals and monitor their progress by producing computer profiles every 3 months. They also monitor their reviewers, some of whom become too zealous, and they may be changed if necessary.

The PROs are now moving into the more complex world of office practice and a pilot study is under way over 2 years studying 80,000 records with outcome measurements. ${ }^{5}$ They are also becoming more interested in quality issues rather than being seen in their original format as cosê̊ control organizations. ${ }^{6}$ The private insurance com- $-\frac{3}{\infty}$ panies seem disinterested in quality issues and $\mathcal{Q}$ almost all their efforts are related to cost contain $-=$ ment.

\section{Conclusions}

Quality assurance has a long history in the US. The technology already in place for billing has made ites relatively easy to collect and analyse quality issues. $\vec{O}$ Whilst activities in the hospitals mirror what is jus $\dot{C}$ starting in the UK, the ability of organizations like PROs to monitor trends in large numbers of doctors gives them the potential to detect where 3 quality is substandard and feed corrective actionis into the educational system. The medical defence companies in the UK have some of this knowledge, but apart from a rather turgid annual report documenting mistakes, have taken little part in medical education.

Hospitals in the UK also need a more clearly defined mechanism to deal with the small minority of doctors who are performing inadequately, $\mathbb{O}$ preferably through educational means, and doctors need to be humble enough to realize that during a professional career that may span ovepo $4 \cdot \overrightarrow{0}$ decades they are going to need re-education. Finally, how large a problem do we have? It wis suggested to me by several doctors that about $10 \%$ of medical encounters in the US have a problems with quality - it is unlikely to be less in the UK and

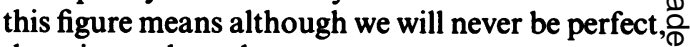
there is much to do.

\section{Acknowledgements}

I would like to thank the National Association of Clinical 3 Tutors for arranging my trip and Wyeth Pharmaceuticalsifor sponsoring it. In particular my thanks go to Dr Gene Meyer, Director of the Area Health Education Centersi Program, North Carolina for his hospitality and also to 3 many other physicians and health care professionals in the State.

\section{References}

1. Califano, J.A., Jr. America's Health Care Revolution. Who lives? Who dies? Who pays? Random House, New York, 1986.

2. Callahan, D. Rationing medical progress. The way to affordable health care. N Engl J Med 1990, 322: 1810-1813.

3. Scovern, H. Hired help. A physician's experience in a for-profit staff-model HMO. N Engl J Med 1988, 319: 787-790.

4. Dans, P.E., Weiner, J.P. \& Otter, S.E. Peer review organizations. Promises and potential pitfalls. N Engl J Med 1985, 313: $1131-1137$.

5. Ellwood, P.M. Outcomes management. A technology of patient experience. $N$ Engl J Med 1988, 318: 1549-1556.

6. Lohr, K.N. \& Schroeder, S.A. A strategy for quality assurance 0 in Medicare. $N$ Engl J Med 1990, 322: 707-712. 